\title{
'A little heaven in hell': the role of a supervised injection facility in transforming place ${ }^{1}$
}

\author{
Ehsan Jozaghi ${ }^{2}$ \\ School of Criminology \\ Simon Fraser University

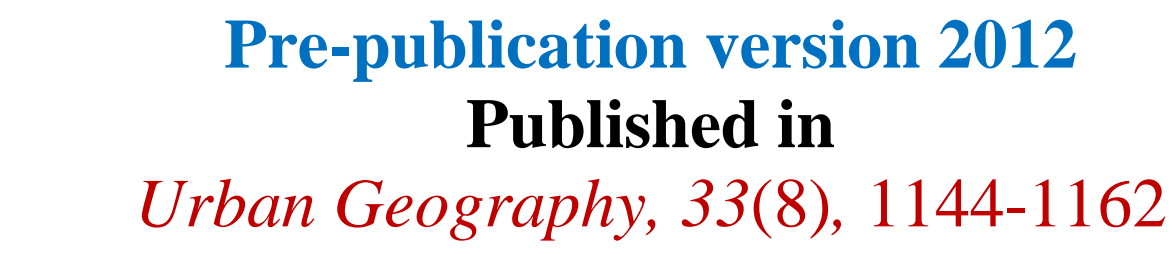 \\ To link to this article: $\mathrm{https://doi.org/10.2747/0272-3638.33.8.1144}$
}

\footnotetext{
${ }^{1}$ I would like to thank Dr. Martin A. Andresen and Dr. Ted Palys for their help and expertise that has proven invaluable for this study.

${ }^{2}$ School of Criminology, Simon Fraser University 8888 University Dr., Burnaby, BC, Canada V5A 156 email: eja2@sfu.ca
} 


\section{Abstract:}

While numerous studies on InSite (North America's first supervised injection facility) have been published in leading international journals, little attention has been given to the potential role that InSite has played in changing the culture of drug use and transforming place in the Downtown Eastside. The present study was conducted to explore the transformative role of InSite in the lives of injection drug users (IDUs). Semi-structured qualitative interviews were conducted with a purposively chosen sample of six IDUs who were attending InSite. Interviews were transcribed verbatim and analyzed thematically using NVivo 8 software. Participants' narratives indicate that attending InSite has had numerous positive effects in their lives, including changes in sharing behavior, improving health, establishing social support and saving their lives. Furthermore, attending InSite has been particularly effective in creating a unique microenvironment where IDUs are increasingly identifying InSite as their community center. [Key words: supervised injection facility, culture of drug use, Downtown Eastside of Vancouver.] 


\section{Introduction}

Vancouver, Canada with 642,843 population is ranked as the most liveable city in the world and recently hosted the 2010 Winter Olympics (Cayne \& Associates, 2006; Chai, 2011). However, the city's Downtown Eastside (DTES) neighbourhood was also recently highlighted in a United Nations report as the most troubled and drug infested neighbourhood in North America (Small et al., 2006). As Philip Owen, former mayor of Vancouver, stated: “The Downtown Eastside is ... a topsy-turvy world where needle-scarred addicts shoot up in the shadows [of the

alleys] (see Figure 1). The open-air drug market flourishes mere blocks from the police station; where some homeless prefer to bunk down in a vomit-drenched doorway rather than a buginfested room; and where people stumble and shuffle through streets, frantically picking at an imagined hole in their body or arguing with voices only they can hear" (Chan, 2009, p. A5). Many people have gone from struggling with mental illness to becoming drug addicted, very sick, and often homeless. As Larry Campbell, another former mayor of Vancouver describes:

When we deinstitutionalized, we promised [the mentally ill] ... that we would put them into the community and give them the support they needed ... We said we'd put them in the community with care and help. Instead, we gave them medication and a bus ticket, and they came to Vancouver. Then they started self-medicating with alcohol and, later, illegal drugs ... we created a "disgusting ghettoization" of the severely mentally ill in areas such as Vancouver's Downtown Eastside (Campbell et al., 2009, p. 83).

\section{<Insert Fig. 1. Here>}


The shortcoming in the system became more severe when the Federal Government in Canada decided it could no longer support social housing programs in order to cut spiraling deficits (Campbell et al., 2009). The situation was a disaster for Vancouver where the number of units renting at or below the welfare allowance dropped from 9,100 in 1992 to 7,800 in 1998 (Campbell et al., 2009). As a result, with little support, many low income individuals not only became homeless, but they became the targets for "friendly" dealers when the drug prices fell (Campbell et al., 2009). According to Dooling and Rachlis (2010),

other factors have also contributed to Vancouver's concentration of people with addiction. A disproportionate number of residents are Aboriginal people, many reeling from unhappy lives on unhealthy reserves. The city's relatively warm climate has attracted many people with addictions from other provinces. Finally, Vancouver is a major Pacific port, through which substantial quantities of illicit drugs pass (p. 1440).

The ten-block area of the DTES (see Figure 2) is the poorest urban area in Canada, and have become home to approximately 4700 of Vancouver's estimated 8000 IDUs whose lives are further marked by extreme poverty, mental illness, lack of housing and social support (Kerr et al., 2003; Mass et al., 2007).

\section{<Insert Fig. 2. Here>}

Consequently, places such as the DTES that have a denser population of IDUs than any other neighbourhood in Canada compound the risk factor for HIV and overdose deaths when individuals with similar lifestyles and activities are likely to associate with one another (Maas et al., 2007). In essence, as result of sharing needles in shooting galleries (see Figure 3), the DTES has an HCV rate of just below $70 \%$ and an HIV prevalence rate of 35\% rivalling third world countries such as Botswana (Chan, 2009;

Maas et al., 2007; Wood et al., 2004). As a result, the DTES neighbourhood and people who live there 
might account for morbidity variations not seen in any Western community, distinguishing itself as a 'landscape of despair' (Gesler, 1992). Many IDUs are still drawn to the area because of availability drugs and the growing level of street level health services (Smith, 2003).

\section{<Insert Fig. 3. Here>}

In order to reduce the community, public health and fiscal impacts of injection drug use, North America's first supervised injection facility (SIF), known as 'InSite', opened its doors on September $15^{\text {th }}, 2003$ in Vancouver's DTES (see Figure 4). The opening of the SIF in Vancouver was based on the belief that "people addicted to injection drugs would have improved health and social outcomes if they could inject drugs in an environment that is clean and medically supervised" (Small et al., 2006, p. 79). Figures from all levels of government (municipal, provincial and federal) came together to support the opening of North America's first SIF since a national task force in 2000 had already recommended to the provincial and federal government that a medical research project such as a SIF was necessary in Vancouver (Campbell et al., 2009).

\section{<Insert Fig. 4. Here>}

InSite offers a space for IDUs to use previously obtained illicit drugs under medical supervision of health professionals in a hygienic and safe environment (Fast et al., 2008). Within InSite, IDUs are typically provided with sterile injection equipment (e.g., insulin syringes with attached needles, bottles of sterile water for injection, latex condoms, alcohol swabs, disposal boxes and spoons), food, and coffee. Also available are emergency care in case of overdose, and referral to addiction services (Wood et al., 2005).

A typical day in InSite (7days a week: 10-4 AM) will see 600 supervised injections within the small 12 individual booths for injections (Kerr et al., 2007). With over 8000 unique registered users, InSite provides an unparalleled setting for research and evaluation (Webster, 2008). Many of the 
scientific evaluations of InSite have been published in leading international journals, reporting an array of positive community and ground breaking public health benefits.

However, the majority of such studies have been quantitative in nature, and have failed to critically examine the lives, stories and circumstances of IDUs who have used and continue to use InSite. Further, we know of no studies that have accounted for the transformation of landscape, such as the DTES, for IDUs with regard to SIF as a therapeutic place, powerful enough to reshape meanings, identities and values. In fact, recent micro risk environment research has suggested that risk perception and behaviour is a product of peer group and social influences, values and the local neighbourhood and context in which IDUs live (Rhodes, 2002). In addition according to Moore and Dieze (2005), creating enabling environments such as InSite "provide more conductive settings for the adoption of individual behaviour change to reduce drug related harm" (p. 276). The current study seeks to determine whether there has been a transformative role (culture ${ }^{3}$ of drug use and landscape) in the DTES. And if there has been a change, would this change be powerful enough to reduce drug related harm? The specific research interests are listening to IDUs' views of InSite, determining how InSite's establishment has reshaped values, and considering what can be done to attract even higher percentages of IDUs to the site.

\section{METHODOLOGY}

Beginning in October 2009, participants living in Vancouver who had injected illicit drugs in the previous month were recruited to participate in the study. The Participants were eligible for the study if they had injected illicit drugs at least once in the previous month, were 19 years or older and provided informed oral consent ${ }^{4}$. They received CAD $\$ 10$ reimbursement for their participation at the end of the

\footnotetext{
${ }^{3}$ Culture in this context is defined as "the process of negotiating meaning with respect to constantly changing implicit and explicit values that underpin the moral fabric of social action" (Small et al., 2006, p. 73).

${ }^{4}$ Written consent deemed to be impractical due to confidentiality.
} 
semi-structured interview. This study was approved by the Simon Fraser University's Research Ethics Board.

This qualitative research draws on a small sample of six participants and does not claim to do the work of large quantitative studies in which representativeness and generalisability are defined. Nevertheless, themes are identified and these are recognized as meaningful whether or not they apply in all cases ${ }^{5}$. In fact, according to Small (2009) "there is a place for a small interview study to make meaningful contribution to knowledge ... [since] rare situations are often precisely what the researcher wants" (p. 18). In effect, the purpose in qualitative study such as this one is to understand the case, not to generalize from it (Burawoy et al., 2000). Further, the focus is to uncover the mechanism and the process (Small, 2009).

Interview participants were recruited along Hastings Street through a key informant as no permission was granted to recruit participants inside InSite. The key informant was an Iranian IDU whom I met accidently when I saw him arguing in Farsi with another IDU. Since Farsi is my first language I was able to talk to him and establish the trust and rapport necessary to launch my interviews. Over the next two weeks he was not only the gatekeeper into the world of IDUs, but he agreed to help me to recruit IDUs that he knew. In other words, I used him as my key informant and guide during selection of participants and referrals.

Interviews took place where the participants felt comfortable. The key informant helped me to establish a rapport and trust among IDUs. In fact, all IDUs not only revealed their HIV/HCV status, but they spend between 15-20 minutes during the interviews answering my questions. There were no refusals of invitations to participate in the study. All the participants were only interviewed once. In order to maintain confidentiality, all names used in this paper are pseudonyms. The key informant also

\footnotetext{
${ }^{5}$ See Grbich (1999) for discussion of these issues.
} 
proved to be instrumental in guiding the sampling selection based on participants' drug of choice, years of injection, race and gender as outlined in table 1.

\section{<Insert Table 1 About Here>}

The open ended, semi-structured interviews were facilitated through the use of an interview guide. The Interview guide encouraged discussion about SIF, the impact of SIF in upon their behaviour, and elicited suggestions related to the ways it can be improved. The questions were chosen based on the ideas and suggestions of numerous peer reviewed journals and is provided in the appendix. The themes that I followed throughout the interview in Vancouver were along the following four paradigms: (1) experience at InSite; (2) their relationship to staff; (3) changes that they have noticed in their behaviour and the Downtown Eastside; and (4) an open discussion about anything raised during the interview.

Their responses were audio recorded and later transcribed verbatim. The content of interviews was reviewed, and all text segments were subsequently subjected to a thematic analysis using NVivo 8 software. Initially an open coding method of searching for similar words or repeating phrases was employed. Twenty five coding categories emerged. Silverman \& Marvasti (2008) warn of the tendency for coding schemes to become "powerful conceptual grid[s] from which it is difficult to escape" (p.225). Therefore, each coding category was reviewed again at a later date, this time using the key themes as coding categories. Each coding category was reviewed independently for latent meanings and common ideas. The main thematic analysis focused on the social processes and experiences that were reported to influence broader and more long-term behavioural changes within IDUs. Validity is an important concept in both quantitative and qualitative research that was considered in this analysis. Validity is defined by Hammersley (1990) as, "truth: interpreted as the extent to which an account accurately represents the social phenomena to which it refers" (p.57). In order to maintain validity in this 
research and avoid "anecdotalism", quotes were considered both in the context of the interview and as a standalone representation of a theme.

\section{Findings}

The results below are based on the sample of qualitative interviews conducted with six purposively chosen participants. Excerpts from the qualitative interviews are presented below in order to illustrate the central themes that emerged in the cross case analysis. These themes included provisions such as: saving lives, changes in sharing behavior, improved access to care and unique microenvironment. Although data was analyzed from each participant independently, considerable overlap was observed across these thematic areas after using NVivo 8 software, indicating overall positive changes in the lives of those who use InSite.

\section{Saving lives}

The most common narrative offered by the study participants was that InSite is saving lives. In fact, all the participants have experienced overdose or have seen people overdose at InSite. In the case of the first participant, Niki, the overdose experience at InSite is a recent one: "InSite is safe. I overdosed not long ago, maybe a few weeks ago. And they saved my life. I have seen others overdose there and they saved their lives too. There've been no deaths. Overdose is really painful. You don't know you're overdosing till you come to it, that's when they Narcan ${ }^{7}$ you to get your heart going again. It's fucking painful. Y'know ... scary",

\footnotetext{
${ }^{6}$ Anecdotalism is defined as taking lone entertaining instances to be representative of a consistent theme (Silverman \& Marvasti, 2008).

${ }^{7}$ Narcan or Naloxone is a drug used to counter the effects of opioid overdose (Old and Swagerty, 2007).

${ }^{8}$ All quotes in this paper are verbatim to accurately reflect language usage by IDUs.
} 
This sense of fear and uncertainty associated with overdosing was also present in the narratives of other IDU's. In effect, IDUs who used InSite have come to associate outside injection with a substantial risk of death and arrest possibilities which they are simply not willing to take. Again, the common underlying theme was staying alive. For example, as Ayatollah stated:

When junkies like me want to inject, we need to find a secluded corner like a bathroom or behind a coffee shop. We're always trying to hide from cops ... In the alley, I might even do a bigger whack because I might lose it if cops come around. Sometimes I used to do it behind a dumpster. Nobody is going to know you're there ... So this is a big issue for me because once you OD, you're done. In addition, many participants noted that injecting at InSite reduced their anxiety because they did not have to inject under the watchful eyes of the public. Participants associated public injection with unpleasant past experiences such as theft and unhealthy behaviour. In light of their memories, InSite was described as a safe and trusting environment that saves lives. In fact, as Alex described: "Seeing people using the rain pot holes on the street for their injection or sharing, or being robbed had a big influence on me. Big time really. Think about it, I've seen it by my own eyes, they're gonna wait' til you drop; then you'll get 'duged' [robbed] ... But at least at InSite I feel like someone would dial 911 and help me ... Since then, I decided to always do it here ... at least I'm safe here".

Furthermore, the knowledge and the rapid response of the staff and nurses to overdose was another potential reason for many IDUs to rely on InSite for all of their injection needs. The urgency of the responding nurse and the care during the overdose conveyed the message that their lives are valuable. This in turn, affected their self evaluation. In addition, InSite has contributed to changes in values of IDUs. Many of the IDUs asserted that they feel safe, secure and appreciate that their lives are valued at InSite. Although such values are hard to quantify, the immediate and long term consequences 
may serve to better their lives. Hence, according to Ayatollah: "When you see how nurses are running to save lives, you can imagine yourself being saved if you ODed too. In fact, I can remember the last overdose. The guy suddenly dropped. Boom, right away, the nurses Narcaned him. One nurse was holding his hand, telling him that he was going to be Ok ... Or how they treat you as human beings; it does make you feel good. When I come to InSite, I don't feel that I'm a junkie anymore". It is situations like these that suggest InSite may play a substantial role in managing and reducing overdose within the street based drug scene of the Downtown Eastside (Kerr et al., 2007). Also, it is situation like these that have conditioned the growing empowerment and enhanced self image of participants over the past six years

\section{Changes in sharing behaviour}

The previous discussion has outlined, albeit implicitly, some of the ways that InSite has contributed to reduced mortality incidence due to overdose and increased sense of security during an injection. However, according to Alex, InSite has done more than save lives; it has modified the drugusing environment of the Downtown Eastside, including the social dynamics within them:

Before InSite opening, I can remember that IV dope [intra-venous drug] was everywhere. Ok, Its start to being an epidemic ... And you could walk the streets and see people fixing [injecting] ... They were leaving their rigs [needles] everywhere. The HIV was growing rapidly as people were sharing rigs ...There were people ODing [overdosing] everywhere ... Junkies were dropping like flies. And all of the sudden we heard about InSite ... all of us are going wow; this is too fucking good to be true. So over the long run, this place has fixed the Eastside. It has cut down on people fixing [outside], ODing, or getting diseases. 
For instance, Tyndall et al., 2006 and Wood et al. (2004) have found that the opening of InSite was independently associated with improvements in several measures of public order, including reduced public injection, drug use, public syringe disposal, and to enhance HIV prevention. The accounts of these interviewees help explain why this is the case.

The participants reported that the provisions of sterile syringes, the ancillary injecting equipment and safer injecting advice by nurses has served to reinforce permanent adoption of safer injecting practices. As Ayatollah insisted: "Why would I share? Nurses have taught me so much about disease transmission that I'm scared to share again. Also, there is so much supply of rigs and stuff like that; sharing is the last thing on my mind. I can even take a box of needles if I wanted”. As a result, most of those who described injecting at InSite with clean needles would not be willing to share again. In other words, once safer injecting habits are established within InSite, it becomes more likely that this safer injecting technique will be extended beyond the facility as well. For example as Alex noted: "Everyone that I know comes to InSite. I have used rigs outside, only because they were full and I was fucking dope sick. But that's rare. I tend to wait. I do feel strong about disease and all of that stuff. So I've not shared with anyone since coming here. It's a habit of being safe everywhere".

Importantly, HIV positive IDUs who have been coming to InSite also tend to follow safe injecting habits outside of the facility. For example, as Niki asserted: "Let me put it to you this way, I got HIV by sharing needles. I don't want to give it to somebody else. But when you are high you don't realize it. But I wouldn’t share again. [Interviewer: Really? Even if you were low in cash?] Of course not [angry]. I already told you that I'm HIV positive. I do not want to give it to someone else".

Another factor that appeared to signify the direct influence of InSite on those who rely on its services was an awareness of 'having something to lose'. In effect, these individuals view themselves as people who are healthy and hopeful of the future. As Ayatollah stated: 
InSite also has a social effect too. Because people that [don't] come here have a different mentality. ... They're more fucked. They just wanna get high. They don't give a fuck. They want to be wasted. If that means using somebody else's needle fucking right they'll do it ... Most of them have got Hep C and that, so they think they're done anyways ... I mean they can catch HIV too ... That's the thinking of those people that I call 'alley junkies'. That's what you're dealing with ... At least I am not catching diseases.

The reassurance of health seems to act as a positive reinforcement for relying on InSite. This change of behaviour outside of InSite — in accordance with the theory of Planned Behaviour ${ }^{9}$ — is thought to be determined by the intention of not reusing another IDU's syringes or the difficulty one perceives towards adopting or maintaining a given behaviour (Côté et al., 2006). It appears IDUs who visit InSite perceive themselves as being in control in situations where high risk sharing is likely outside of SIFs (Côté et al., 2006). Comparing themselves to those who do not come to InSite reinforces their differences from 'alley junkies'. In other words, many of the IDUs' accounts seem to indicate that InSite plays an important role in initiating labelling, membership, and shared values, a critical step toward a collective identity and changing the culture of drug use.

\section{Improved access to care}

In addition to the improved changes in behavior and shared collective identities described above, InSite is often a client's first point of contact with the health care system. Most participants described having accessed a variety of services at InSite. These primary care activities, according to participants,

\footnotetext{
${ }^{9}$ Theory of planned behaviour states that "the proximal determinant of behaviour is the intention to act. The intention, in turn, is influenced by the attitude towards the behaviour, subjective norm, and perceived behavioural control" (Hardeman, 2002, p. 124). See Ajzen (1991) for more information regarding the theory of planned behaviour.
} 
include immunization, screening, diagnosis and treatment. Many participants also reported they were able to access much needed support at InSite for chronic wound care. Health care staff and nurses at InSite are familiar with various injection-related infections. In addition to care, staff at InSite provide funding and referrals, facilitating further medical attention. For instance, according to Dan: Here at Insight, we are checked every six months for HIV and Hep C. They also have the same day result HIV testing at InSite. I've got both my H1N1 shot and a regular flu shot there. Also for the past five years I had serious abscesses on my lower leg, and they changed my bandaging for five years ... And when I needed my antibiotics shots, they actually provided the funding for me to get to and from the hospital.

These findings are very similar to an earlier study by Small et al. (2008) that reported that InSite "enables contact with the healthcare system and thereby helps to facilitate the management of injectionrelated infections" (p. 159).

Many participants' accounts seems to suggest that they were able to get much needed help for their complex needs, which often included mental illness, homelessness, detoxification and support. Counselors and support staff are regarded as providing critical social support at the time of crisis. The provision of health care and support are seen by participants to be highly effective models of care for IDUs. For example, one of the participants referred to it as the 'clinic for junkies'. Participants also appreciated that they are able to access various forms of care at one location calling it a 'supermarket for junkies'. Participants' perspectives indicate that the facility's integrated health Model (where addicts have access to detox (withdrawal management), counselors, primary care and Support) is particularly effective in creating an atmosphere of support. Moreover, most participants felt that InSite was like a family. As Niki described: 
Well, I come to InSite for few reasons. First you don't catch disease. Second if you OD you aren't going to die. Third everything is available. You might not have alcohol, water or rig; but when you go there you can get whatever you need for free. It's like a 'supermarket' for junkies ... I also like the fact that you can talk to counsellors and nurses for support. They've helped me to get rooms at shelters before ... They've always been there for support ... When I found out I was positive few months ago [crying] ... they were there for me ... InSite is like a family to me ... I've gone through detox upstairs ... It's like a clinic for junkies. Similarly, Wood et al.'s (2006) study reported that "contact with addiction counsellor[s] was among the strongest independent predictors of more rapid entry into a detoxification program" (p. 2513). For many InSite users, "the availability of immediate and easily accessible care translates into receiving health care services they would otherwise do without" (Vancouver Coastal Health, 2007, p. 4).

Participants also described InSite as a unique educational and life-changing setting that has impacted their injecting practice and reduced occurrence of injection-related overdose or disease. In addition, according to Dan, staff at InSite were relied on for social support and educational advice: They teach us how to properly inject ... It's changed my way of thinking, the way I use drugs, the amount I use ... Before InSite, I didn't care whether I OD[ed] or not. I didn't care whether I died. Now I am starting to care about myself more, I have more self respect. Just because of InSite. Now that I've somewhere to go and someone to talk to about it, I don't use as much.

IDUs' narratives here provide reassurance that InSite's services and staff may be extremely effective not only in improving medical access for treating injection-related infections, but they are equipped to provide education and counselling. Fast et al. (2008) also reported that InSite "has been particularly 
effective in transmitting educational messages targeting unsafe and unhygienic injection practices to a population of active IDU” (p. 1). InSite represents a unique micro-environment that can facilitate the reduction of numerous drug related harms through education, counselling and access to care.

Further, participants' accounts seem to indicate that staff and nurses at InSite have gained awareness into holistic strategies and approaches that go beyond simply providing care. In effect, the staff have been able to create dignified, caring and trusting bonds that build foundations for change through personal empowerment. For instance, according to Lisa:

I think the way they treat us is great. I personally think their act of kindness is changing lives down here ... They always talk to me and respect me ... They're like my family ... The staffs at InSite tell us that we are not losers. There is no judgment there. I'm not judged or mocked for what I am. They help us to build character.

In effect, the close bond and the relationship that exists between the staff and IDUs at InSite has facilitated more than 2,000 referrals to addiction services, with 800 of these referrals to addiction counselling (Vancouver Coastal Health, 2007). Small et al. (2008) suggests that further benefits may be gained by expanding the contact and relationship between IDUs and staff through increased nursing and counselling care using outreach services. In effect, InSite is a 'therapeutic landscape' in which "environmental, individual, and societal factors ... come together in the healing process" (Gesler, 1992, p. 735). As Conradson (2005) suggests "it is possible that the emotional contours of their visit may have been shaped less by surroundings, and a little more by the relations" and close bonds to the staff at InSite, enabling better health services (p. 113).

\section{Unique Microenvironment}


InSite gives IDUs access to primary care, counselling, syringes, and ancillary injecting equipment. However, research participants also viewed it as something of a 'refugee camp' where distressed IDUs could seek relief and support because it provided a place for many participants to relax, socialize and unwind, thereby enabling them to maintain control over their lives. According to Alex: After injecting at InSite, I don't want to leave. This place is great. It's clean ... When I leave the site, it's again back to the same shit hole ... The air is fucking filthy. You can smell death and diseases down here ... InSite is like a little heaven in hell. Also ... I go there to meet my friends and socialize.

As these quotations illustrate, InSite is not simply an injection site. Rather, it is an integral part of a comprehensive continuum of addiction service that tries to alleviate their misery and pain. For people with prolonged drug addiction, InSite is "the first rung on the ladder from chronic drug addiction to recovery; from being ill to becoming well" (Vancouver Coastal Health, 2007, p. 1). Consistent with those intentions, many IDUs view InSite as their community center. For them, InSite is a place where all IDUs gather for support and acknowledgment. It is a place that gives IDUs a collective voice and identity. In other words, InSite is a place where IDUs show the rest of the society that they exist. As Dan suggests:

InSite is more than a place to get a fix. They've [the staff] build relationships with everyone. It's like a home away from home. It's like a support center for junkies. The staff listen to you; they actually hear you and feel what you're going through. And I think an increasingly large numbers of people are coming there to get acknowledged.

Duff (2009) also suggests that enhancing local networks, increases belonging and "connection to place" and reinforces the "culture of care". Participants who have been coming to InSite for a few years felt 
empowered to help others, having seen the transformative power of InSite (either through counselling, social support, or overdose emergency care) and craving for change within their own community. Many participants had become counsellors for their own peers, advocating and encouraging InSite use at every opportunity. For example, Dan stated:

I can socialize with other drug users and help them ... We try to get in touch with junkies that never come [to InSite]. In fact, I've convinced a few people this year to come. Because the first thing we say is: Hey! You're going to do some dope, if you OD out here we're gonna take your shoes and steal all your money. But if you OD at InSite, you've got nurses that can bring you back to life, guaranteed ... We are promoting InSite every chance we get.

Zapka et al. (1993) also found that social support, social net works and social influence of IDUs with their peers and friends improved drug use behaviour. This dramatic advocacy for Insite and on behalf of other users (by participants who once injected and shared outside) can be better understood through symbolic interactionism (Sandstorm et al., 2006). Symbolic interactionism, associated with George Herbert Mead and Robert E. Park is a paradigm that dominates social science by emphasizing interaction, meaning and human behaviour at the center of our understanding (Sandstorm et al., 2006). The most important aspect of this paradigm in the realm of InSite is related to the notion that "people are conscious, self-reflexive beings who shape their own behavior when interacting with others" (Sandstorm et al., 2006, p. 9). An implication of this perspective is that participants' involvement through interaction and role-taking at InSite can contribute to IDUs' understanding of their roles within their own community. And indeed, many participants identified themselves as safety and education ambassadors within the DTES. In fact, as Alex described: 
I always carry extra rigs and shit like that to give to other junkies. When I see a few of them doing it in the alley, I'd tell 'em about InSite. In my experience, they tend be shy. I tend to call them 'closet users' [laughs] ... I can recall, few years back, that I convinced one of the junkies from the street to come here. I walked with her and helped her to register. She was very scared. Now she is a regular user Their new roles as a result of self-empowerment have the potential to mediate patterns of infectious disease and mortality, and eventually change lives amongst the most marginalized IDUs.

\section{Discussion and Conclusions}

This study initially set out to examine the transformative role of InSite in the lives of IDUs. The findings revealed a positive change in many respects: InSite prevents drug overdose deaths, reduces HIV risk behavior (e.g., sharing needles), and increases access to nursing and other primary health services. Furthermore, many of InSite's clients in this case study have come to view InSite as their family and rely on the social support provided at InSite. Moreover, InSite provides a space of dignity that was otherwise unavailable. Although geographers have rarely thought of sense of place as important in studying health care (Gesler, 1992), it is easy to see how InSite has been therapeutic place for many IDUs who are increasingly relying on its services. In effect, InSite is a 'therapeutic landscape' in which “environmental, individual, and societal factors ... come together in the healing process" and behavioural change that reinforce the culture of care (Gesler, 1992, p. 735). InSite as therapeutic place has also become a community center where enhanced local networks increase belonging and "connection to place".

These positive outcomes are consistent with results of previous research, despite the different methodologies employed. For example, Milloy et al.'s (2008) study concluded that InSite has prevented 12 deaths per year despite over 1000 overdose events. In addition to overdose, Petrar et al.'s (2007) 
evaluation documented numerous positive behavioural changes very similar to the current study. For instance, it reported that clients have fewer rushed injections, fewer outdoor injections, and fewer incidents of unsafe syringe disposals. Similarly, keeping with this study's findings in regard to established support, the study by Lightfoot et al. (2009) reported that nurses, peer workers and staff at InSite have been successful in developing dignified, caring and trusting relationships. These relationships in most cases have led to increase referrals to detoxification programs and long term health services (Vancouver Coastal Health, 2007). The qualitative methods used in the current study offer a glimpse into some of the social dynamics InSite has created among IDUs that lie beyond those aggregate findings.

The current study further reveals that the impact of InSite as a therapeutic place goes beyond bottom-line reductions in deaths and dirty needles to having effected significant transformations in participants' roles and behaviours. In effect, the same way that Smith and Easterlow (2005) used the qualitative approaches to understand and protect public health, the current study examines the various positive therapeutic accounts of IDUs to demonstrate a cultural change in drug use and behavioural change that ultimately reduces drug related harm. DTES is a changing neighborhood that may seem to transition to something entirely different (Mikelbank, 2011). In other words, the establishment of InSite was culturally momentous, shaped by many unique micro-environmental factors that have conditioned the growing empowerment and enhanced self image of participants over the past six years. IDUs' selfefficacy and empowerment is built upon their enhanced access to health care, social services, counselling and support.

The new self image, in turn has helped the participants to alter the structural and cultural dimensions of power relations that manifest a disproportionate suffering (Fairbairn et al., 2008). Further, there are indications that the DTES is beginning to be transformed as those participants who 
increasingly rely on InSite have gradually become active within their community, trying to alleviate misery and improve lives in the DTES. In fact according to Curtis and Mills (2011) the built environments like InSite have an effect on variety of social outcomes as diverse as public health. This paper identifies participants who strive to better their peers' health and their communities' self image as educational and safety ambassadors. In effect, those who increasingly rely on InSite have gradually become active within their own community, trying to alleviate misery and improve lives in the DTES. The cultural shift in drug use reported here is a new phenomenon, something that has not been reported by previous research. However, it seems that the DTES is still 'hell' and more data is required to determine how far the DTES has been transformed.

Despite the noted findings, the current study has several limitations that should be acknowledged. The most significant can be attributed to data limitations. While many provisions related to perspectives, experiences and values of IDUs are reported, triangulation was limited due to access and the time spent in the field. For example, I would have preferred to supplement many of the interviews with more observations, specifically in regard to interactions within the facility and at night. Although I would have liked to conduct a few interviews at night and conduct observations, due to safety reasons I had to rely on daytime interviews only. This could have ultimately hindered the range of variations within the study because very little is known about perspectives and demographics of those who inject at night.

Secondly, although purposive sampling combined with snowball sampling has shown to be instrumental, additional participants would ultimately be required. In essence, according to Esterberg (2002), "one of the risks of snowball sampling is that participants may be too similar to one another to give ... [us] the diverse perspective ... [we] want" (p. 94). While the key informants was an invaluable contribution who made this study possible, he is nonetheless the keeper of only one gate into a diverse 
DTES community that contains 4700 IDUs (Mass et al., 2007). Therefore, under no statistical definition of 'generalizability' could the responses of the six participants be considered to reflect reliability the condition of IDUs of the DTES despite their varied characteristics.

Thirdly, studying transient populations such as the IDUs in this study is very challenging. This is particularly true when there is a need to return to the setting later for additional follow ups or clarifications. According to McGregor et al. (1998) locations such as the DTES are usually not associated with easily ascertained addresses or access to telephones. Similarly, five out of the six participants were homeless and the remaining participants had no cell phones or internet access. Although I gave participants my phone number and encouraged them to contact me for follow ups, I never received any calls.

Finally, despite my attempts to reduce the social desirability effect—by avoiding leading questions and reminding the participants that there are no right or wrong answers-its influence on participants is unavoidable. Subsequently, some positive responses in regard to InSite can be attributed to the social desirability effect. In addition, recognizing my own subjective bias as an outsider with 'neoliberal' views ${ }^{10}$ about drug use and order—studying a severely marginalized group, with the power to determine what should be recorded - is an important part of recognizing power relationships that are embodied in research. Consequently, the way I directed the conversation, asked the questions, determined what constituted a correct or complete answer, closed the conversation, or paid the participants may have had an influence on what is reflected in this paper. This is particularly troubling because according to Fraser and Moore (2008) this "may serve to obscure and stigmatize rather than to illuminate drug users and their lives" (p. 748). Therefore, it is possible that social desirability effect on participants and the researcher may have influenced the results.

\footnotetext{
${ }^{10}$ See Fraser and Moore (2008) and Fischer et al., (2004).
} 
Based on the findings and limitations of this study, some recommendations for practice can be made. Interestingly, IDUs raised many of these recommendations during the interview. First, IDUs recommended that InSite should be expanded. In fact, the most widely stated reason for not using InSite was related to the long waiting time. For instance, as Shane suggested:

Personally, the main reason is the line-up. Because people go in and they take forever to find their rigs [veins]. Then you got folks who won't leave the table. For God sake, the place only has 12 booths. Hurry the fuck up! And then lots of girls do their make-up or change their clothes. All of these add-up to the line-up.

This finding is very similar to an earlier study by Petrar et al. (2007) that reported 'waiting for an injection' as the most commonly anticipated barrier to access. Further, other IDUs suggested that operating hours of InSite should be expanded to $24 \mathrm{hrs}$ since in their opinion, most of the injections take place in the morning when InSite is closed. As Niki argued: "It is even worse in the morning 'cause you're trying to stay up so you don't get robbed. They should keep this place open 24hrs. Many times I had to go to the alley after they closed. The door is closed at around 3 [in the morning]. Especially if you were working girl like me and had to do whatever you could to get that dope".

Second, any future expansion proposal of the current site should consider mobile supervised injection facilities. Wood et al. (2003) state that IDUs at most risk (such as those who do not attend InSite) can be better targeted. Indeed, according to participant's accounts, IDUs at most risk prefer the first available needle to the first available clean needle provided at the nearby SIFs a few kilometres down the alley; this is particularly true if they are 'dope sick'. As Shane explained:

I think people tend to share when they don't have a rig or they're high ... You can share when you're dope sick too ... When you're 'dope sick', you lose your bowel movement, you start puking ... It hurts. So you will do anything to get the 
fix, even if you have to share, sometimes you've got no choice. I think that's how I got HIV.

In other words, to reduce physical discomfort, IDUs will sometimes use drug residue from other users' equipment with a disregard of the users' HCV/HIV status (Stein et al., 2007). Through a good site selection, the most marginalized IDUs (those who are HIV positive, homeless, and most likely to share needles rather than travel to the SIFs) are targeted, so that the program would prevent an even greater number of HIV cases.

In summary, this study illustrates that SIFs may hold multiple meanings, as many IDUs pointed out earlier. In their eyes, Vancouver SIF is a unique micro-environmental intervention that not only saves lives, or reduces the social suffering they face on a daily basis, but it also offers a glimpse of hope at the end of the tunnel. As Alex stated, "InSite is like a little heaven in hell." The findings in this study are in keeping with more than 30 peer reviewed studies that have shown that InSite has numerous positive provisions. Furthermore, this study's qualitative data also indicate that InSite's positive health and social effects within the participants over the past seven years may have contributed to a cultural transformation where IDUs — who have attended InSite— have become safety and educational ambassadors within their own communities. Ironically, however, the operation and scientific evaluation of the Vancouver's safe injection site, has been challenged by Canada's conservative federal government publically in media and in courts (O'Connor, 2009; Wood et al., 2008). However, Supreme Court of Canada on September 30 ruled in favour of InSite to remain open (Canada [Attorney General] vs. PHS Community Services Society, 2011 SCC 44). Therefore, demonstrating that InSite's positive impacts contribute to a politically and socially charged discussion would encourage other communities to take a bold step towards protecting their most marginalized and vulnerable people. In fact, as Niki proposed, "there should be one InSite opened in every large city". 


\section{References:}

Ajzen, I., 1991, The theory of planned behaviour. Organizational Behaviour and human decision processes, Vol. 50, 179-211.

Burawoy, M., Blum, J. A., George, S., Gille, Z., Gowan, T., and Haney, L. et al., 2000, Global ethnography. Berkeley: University of California Press.

Campbell, L., Boyd, N. and Culbert, L., 2009, Vancouver's Downtown Eastside and the fight for its future: A thousand dream. Vancouver, BC: Greystone Books.

Canada [Attorney General] vs. PHS Community Services Society, 2011, SCC 44.

Cayne and Associates Ltd., 2006, Ripples of change: Community capacity in Vancouver's Downtown Eastside. Vancouver, BC: City of Vancouver.

Chai, C., 2011, Vancouver, Toronto, Calgary named among world's most liveable cities. Don Mills, ON: Postmedia News.

Chan, C., 2009, Wanted: New hope on Hastings. The Province, February 2, p. A5.

Conradson, D., 2005, Freedom, space and perspective: Moving encounters with other ecologies.

In Davidson, J., Bondi, L., and Smith, M. (Eds.), Emotional geographies, (pp. 104-116). Burlington, US: Ashgate.

Côté, F., Godin, G., Mercure, S., Noël, L. and Alary, M., 2006, Preventing HIV transmission among marginalized injection drug users: New insight from a Québec city based research. International Journal of Drug Policy, Vol. 17, 411-417.

Curtis, A., and Mills, J. W., 2011, Crime in urban post-disaster environments: A methodological framework from New Orleans. Urban Geography, Vol. 32, No. 4, 488-510.

Dooling, K., Rachlis, M., 2010, Vancouver's supervised injection facility challenges Canada's drug laws. Canadian Medical Association Journal, Vol. 182, No. 13, 1440-1444. 
Duff, C., 2009, The drifting city: The role of affect and repair in the development of "enabling environments". International Journal of Drug Policy, Vol. 20, 202-208.

Esterberg, K., 2002, Qualitative Methods in social research. Toronto, Canada: McGraw-Hill.

Fairbairn, N., Small, W., Shannon, K., Wood, E. and Kerr, T., 2008, Seeking refuge from violence in street-based drug scenes: Women's experience in North America's first supervised injection facility. Social Science and Medicine, Vol. 67, 817-823.

Fast, D., Small, W., Wood, E. and Kerr, T., 2008, The perspective of injection drug users regarding safer injecting education delivered through a supervised injecting facility. Harm Reduction Journal, Vol. 5, 1-8.

Fischer, B., Turnbull, S., Poland, B., and Haydon, E., 2004, Drug use and urban order: examining supervised injection sites (SISs) as 'governmentality'. International Journal of Drug Policy, Vol. 15, 357-365.

Fraser, S., and Moore, D., 2008, Dazzled by unity? Order and chaos in public discourse on illicit drug use. Social Science and Medicine, Vol. 66, 740-752.

Gesler, W. M., 1992, Therapeutic landscapes: Medical issues in light of the new cultural geography. Social Science Medicine, Vol. 34, No. 7, 735-746.

Grbich, C., 1999, Qualitative research in health: An introduction. St. Leonards: Allen and Unwin.

Hardeman, W., Johnston, M., Johnston, D. W., Bonetti, D., Wareham, N. J., Kinmonth, A. L., 2002, Application of the theory of planned behaviour in behaviour change interventions: A Systematic review. Psychology and Health, Vol. 17, No. 2, 123-158.

Hammersley, M., 1990, Reading ethnographic research: A critical guide. London: Longmans. 
Kerr, T., Wood, E., Palepu, A., Wilson, D., Schechter, M. and Tyndall, M., 2003. Responding to an explosive HIV epidemic driven by frequently cocaine injection: Is there a role for safe injecting facilities? Journal of Drug Issues, Vol. 22, 579-608.

Kerr, T., Small, W., Moore, D. and Wood, E., 2007, A micro-environmental intervention to reduce the harms associated with drug-related overdose: Evidence from the evaluation of Vancouver's safer injection facility. The International Journal of Drug Policy, Vol. 18, $37-45$.

Lightfoot, B., Panessa, C., Hayden, S., Thumath, M., Goldstone, I. and Pauly, B., 2009, Gaining Insight: Harm reduction in nursing practice. Canadian Nurse, Vol. April, 16-22.

Maas, B., Fairbairn, N., Kerr, T., Li, K., Montaner, J. and Wood, E., 2007, Neighbourhood and HIV infection among IDU: Place of residence independently predicts HIV infection among a cohort of injection drug users. Health \& Place, Vol. 13, 432-439.

McGregor, C., Darke, S., Ali, R., and Christie, P., 1998, Experience of nonfatal overdose among heroin users in Adelaide, Australia: Circumstances and risk perceptions. Addiction, Vol. 93, 701-711.

Mikelbank, B. A., 2011, Neighborhood déjá vu: Classification in metropolitan Cleveland, 19702000. Urban Geography, Vol. 32, No.3, 317-333.

Milloy, M., Kerr, T., Tyndall, M., Montaner, J. and Wood, E., 2008, Estimated drug overdose deaths averted by North America's first medically-supervised safer injection facility. Plos One, Vol. 3, 3551-3561.

Moore, D., and Dietze, P., 2005, Enabling environments and reductions of drug related harm: Re-framing Australian policy and practice. Drug and Alcohol Review, Vol. 24, 275-284. 
O’Connor, E., 2009, Drugs, harm-reduction and rehabilitation: InSite awaits fate amid controversy. The Province, July 07, p. A9.

Old, J. and Swagerty, D., 2007, A practical guide to palliative care. Philadelphia, PA: Williams and Wilkins Publishing.

Petrar, S., Kerr, T., Tyndall, M., Zhang, R., Montaner, J., and Wood, E., 2007, Injection drug users' perceptions regarding use of a medically supervised safer injecting facility. Addictive behaviors, Vol. 32, 1088-1093.

Rhodes, T., 2002, The 'risk environment': A framework for understanding and reducing drug related harm. International Journal of Drug Policy, Vol. 13, 85-94.

Sandstorm, K., Martin, D. and Fin, G., 2006, Symbols, selves and social reality: A symbolic interactionist approach to social psychology and sociology. Los Angeles, CA: Roxbury Publishing Company.

Silverman D., and Marvasti, A., 2008, Doing qualitative research: a comprehensive guide. Los Angeles, CA: Sage Publications.

Small, D., Palepu, A. and Tyndall, M. 2006, The establishment of North America's first state sanctioned supervised injection facility: A case study in cultural change. International Journal of Drug Policy, Vol. 17, 73-82.

Small, W., Wood, E., Lioyd-Smith, E., Tyndall, M. and Kerr, T., 2008, Accessing care for injection-related infections through a medically supervised injection facility: A qualitative study. Drug and Alcohol Dependence, Vol. 98, 159-162.

Small, M., L., 2009, 'How many cases do I need?': On science and the logic of case selection in field-based research. Ethnography, Vol. 10, No. 1, 5-38. 
Smith, H. A., 2003, Planning, policy and polarisation in Vancouver's Downtown Eastside. Tijdschrift voor Economische en Sociale Geografie, Vol. 94, No. 4, 496-509.

Smith, S. J., and Easterlow, D., 2005, The strange geography of health inequalities. Transactions of the Institute of British Geographers, Vol. 30, 173-190.

Stein, M., Dubyak, P., Herman, D. and Anderson, B., 2007, Perceived barriers to safe-injection practices among drug injectors who remain HCV-negative. The American Journal of Drug and Alcohol Abuse, Vol. 33, 517-525.

Tyndall, M., Wood, E., Zhang, R., Lai, C., Montaner, J. and Kerr, T., 2006, HIVseroprevalence along participants of a supervised injection facility in Vancouver, Canada. Harm Reduction Journal, Vol. 3, 36-40.

Vancouver Coastal Health, 2007, From the ground up: Insite's role in accessing treatment and care. Retrieved October 24, 2009, from the Vancouver Coastal health Web site at http://www.vch.ca

Webster, P., 2008, Court halts closure of Canada's safe-injection site. The Lancet, Vol. 371, 1985-1986.

Wood, E., Kerr, T., Spittal, P., Small, W., Tyndall, M. and O’Shaughnessy, M., 2003, An external evaluation of peer-run "unsanctioned" syringe exchange program. Journal of Urban Health, Vol. 80, 455-464.

Wood, E., Kerr, T., Small, W., Li, K., Marsh, D. and Montaner, J., 2004, Changes in public order after the opening of a medically supervised safer injection facility for illicit injection drug users. Canadian Medical Association Journal, Vol. 171, 731-734. 
Wood, E., Tyndall, M., Li, K., Lioyd-Smith, E., Small, W., and Montaner, J., 2005, Do Supervised injection facilities attract higher-risk injection drug users? American Journal of Preventive Medicine, Vol. 29, 126-130.

Wood, E., Tyndall, M., Zhang, R., Stoltz, J-, Lai, C. and Montaner, J., 2006, Attendance of supervised injection facilities and use of detoxification services. New England Journal of Medicine, Vol. 354, 2512-2514.

Wood, E., Kerr, T., Tyndall, M., and Montaner, J., 2008, The Canadian government's treatment of scientific process and evidence: Inside the evaluation of North America's first supervised injection facility. The international Journal of Drug Policy, Vol. 19, 220-225.

Zapka, J., Stoddard, A. and McCusker, J., 1993, Aids Education and Prevention, Vol. 5, 326356. 
Table 1.-Characteristics Of The Sample Of IDUs Of Vancouver

\begin{tabular}{|c|c|c|c|c|c|c|c|}
\hline Name & Gender & Age & Ethnicity & Drug of choice & Years of injection & Medical condition & City \\
\hline Alex & Male & 47 & Caucasian & Heroin \& Crack & 30 & $\mathrm{HCV}$ & Vancouver \\
\hline Ayatollah & Male & 50 & Middle Eastern & Heroin & 20 & $\mathrm{HCV}$ & Vancouver \\
\hline Dan & Male & 37 & First Nation & Heroin & 10 & HCV \& Abscesses & Vancouver \\
\hline Lisa & Female & 39 & First Nation & Cocaine \& Crack & 15 & Bipolar Disorder & Vancouver \\
\hline $\begin{array}{l}\text { Niki } \\
\text { Shane }\end{array}$ & $\begin{array}{l}\text { Female } \\
\text { Male }\end{array}$ & $\begin{array}{l}30 \\
29\end{array}$ & $\begin{array}{l}\text { Caucasian } \\
\text { Caucasian }\end{array}$ & $\begin{array}{c}\text { Cocaine \& Crack } \\
\text { Heroin }\end{array}$ & $\begin{array}{l}14 \\
13\end{array}$ & $\begin{array}{l}\text { HCV \& HIV } \\
\text { HCV \& HIV }\end{array}$ & $\begin{array}{l}\text { Vancouver } \\
\text { Vancouver }\end{array}$ \\
\hline
\end{tabular}




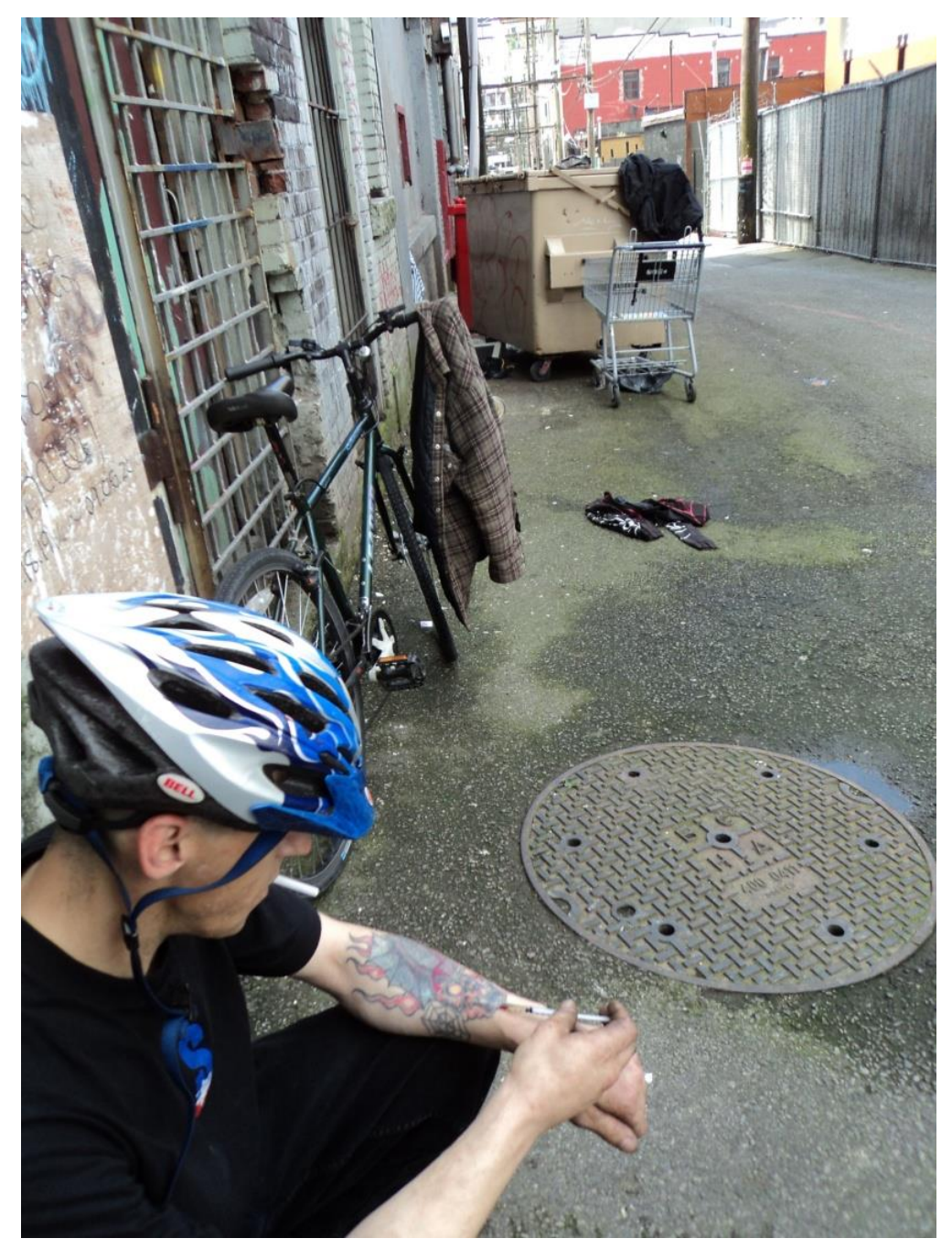

Fig. 1. The DTES contains numerous alleys where IDUs will utilize for their injection. Photo by author. 


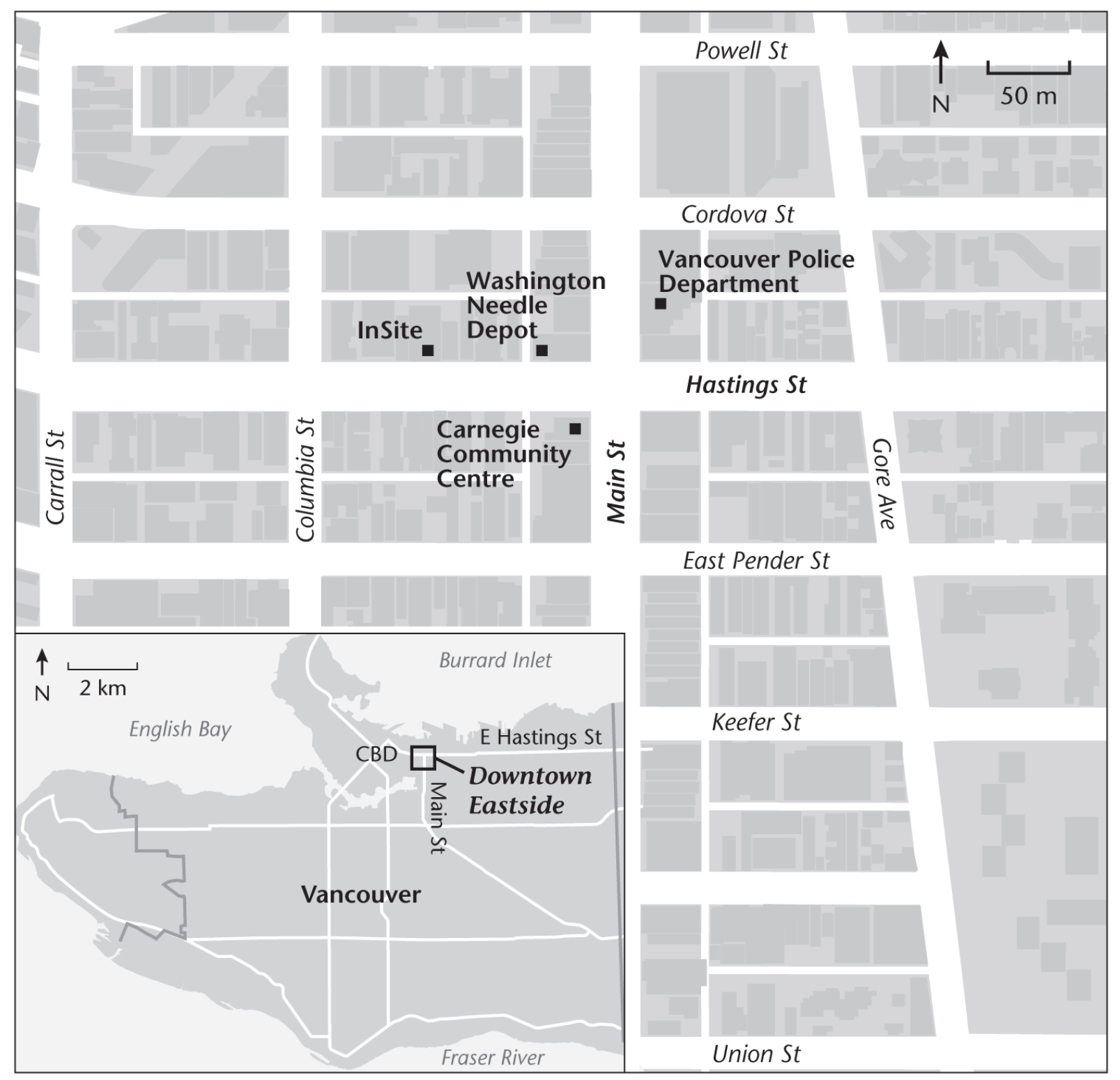

Fig. 2. Map of the DTES of Vancouver 


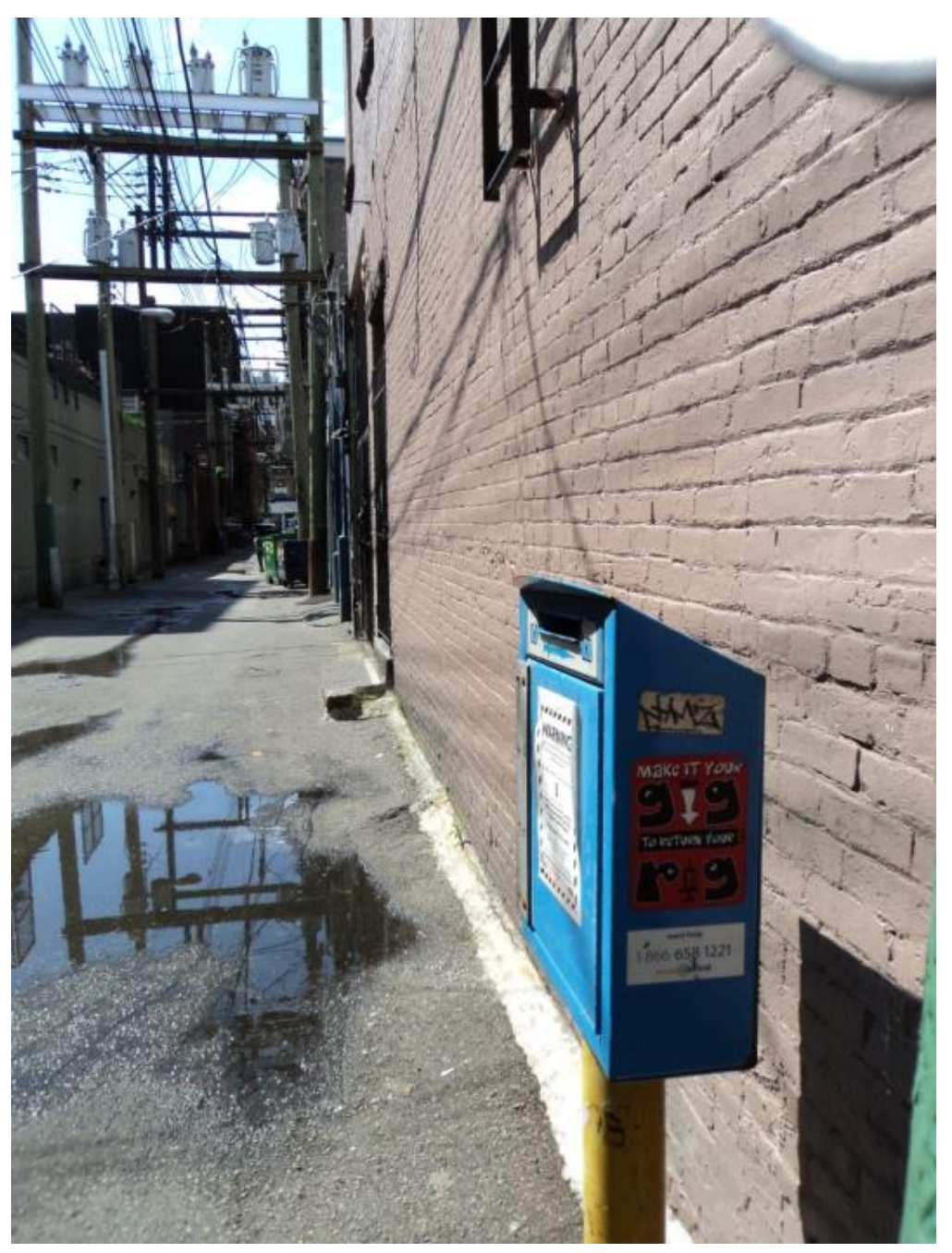

Fig. 3. Needle return boxes are located in all alleys in the DTES containing information about InSite and needle depot to reduce sharing in shooting galleries. Photo by author 


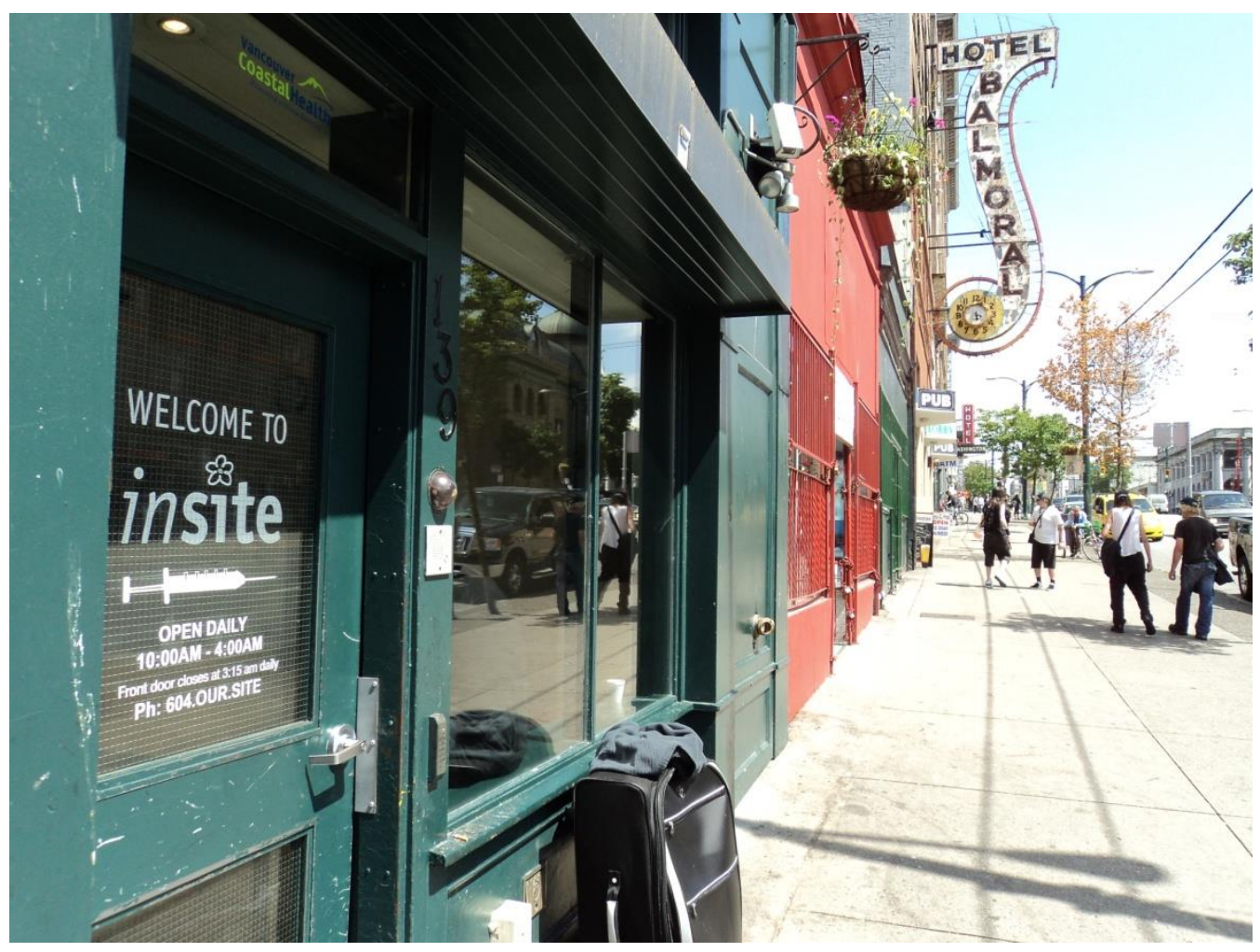

Fig. 4. InSite, North America's first supervised injection facility locted in the heart of DTES. Photo by author 